\title{
Estudo da reação entre tiocianato de amônio e Fe (II) ou Fe (III) utilizando a espectroscopia no infravermelho: um experimento de química prebiótica
}

\author{
Study of the reaction between ammonium thiocyanate and Fe \\ (II) or Fe (III) using infrared spectroscopy: an experiment of \\ prebiotic chemistry
}

\author{
Henrique de Santana ${ }^{1}$; Kelli Esteves Vieira ${ }^{2}$; Cássia Thaïs Bussamra Vieira \\ $\mathrm{Zaia}^{3}$; Dimas Augusto Morozin Zaia ${ }^{{ }^{*}}$.
}

Resumo

\begin{abstract}
A química prebiótica estuda as reações que podem ter desempenhado um papel importante para a origem da vida na Terra. No entanto estas reações devem ser realizadas em laboratórios nas condições que existiram na Terra prebiótica. Os experimentos de laboratório do aquecimento de substâncias em estado sólido podem ser relacionados aos seguintes ambientes prebióticos: resfriamento de lava de vulcões sobre o solo e o impacto de meteoros ou cometas sobre a Terra. No presente trabalho a reação em estado sólido entre o tiocianato de amônio e Fe (II) ou Fe (III) foi estudada. As amostras foram aquecidas a $220^{\circ} \mathrm{C}$ em diversos tempos (6 horas, 24 horas, 7 dias). O principal resultado deste trabalho foi que uma reação de oxidação-redução esta ocorrendo entre o Fe (II) e o tiocianato de amônio, os espetros infravermelhos do produto de reação tiocianato de amônio/Fe (II) mostraram uma banda que é característica do ferricianeto. Os espectros infravermelhos também mostraram neste produto de reação bandas características do tiocianato de guanidina. Portanto, o aquecimento do tiocianato de amônio com $\mathrm{Fe}$ (II) em estado sólido esta formando tiocianato de guanidina e ao mesmo tempo oxidando o Fe (II) para $\mathrm{Fe}$ (III). O produto da reação entre $\mathrm{Fe}$ (III) e tiocianato de amônio é o tiocianato de guanidina. O $\mathrm{Fe}$ (II) e o Fe (III) reagem de forma diferente com o tiocianato de amônio.
\end{abstract}

Palavras-chave: Química prebiótica. Tiocianato de amônio. Ferro. Guanidina

\begin{abstract}
The prebiotic chemistry studies the reactions that could be played an important role on origins of life on Earth. However, these reactions should be carried out under conditions that existed on the prebiotic Earth. The laboratory experiments of heating substances in solid state could be corresponding to the following prebiotic environments: cooling lava from volcanoes on the ground and impact of meteorites or comets on the earth. The present study examined the reaction in solid state between ammonium thiocyanate and $\mathrm{Fe}$ (II) or $\mathrm{Fe}$ (III). The samples were heated at $220^{\circ} \mathrm{C}$ in several different times $(6$ hours, 24 hours, 7 days). The most important finding of this work is that a reaction of oxidation-reduction is occurring between $\mathrm{Fe}$ (II) and ammonium thiocyanate, the infrared spectrum of the reaction product ammonium thiocyanate/Fe (II) showed a band that it is characteristic of ferricyanate. The infrared spectra also showed bands that they are characteristics of guanidine thiocyanate. Thus, the heating of ammonium thiocyanate with $\mathrm{Fe}$ (II) in solid state is synthesizing guanidine thiocyanate and at the same time oxidizing $\mathrm{Fe}$ (II) to $\mathrm{Fe}$ (III). The product of reaction between $\mathrm{Fe}$ (III) and ammonium thiocyanate is guanidine thiocyanate. Fe (II) and Fe (III) are reacting with ammonium thiocyanate in different way. Ke⿻y-wơnds: Prévioutic cheñinistiry. Ammonium thiocyanate. Iron. Guanidine.
\end{abstract}

${ }^{1}$ Professor Associado-C, Departamento de Química-CCE, Universidade Estadual de Londrina.

${ }^{2}$ Bacharel em Química, Departamento de Química-CCE, Universidade Estadual de Londrina.

${ }^{3}$ Professora Associada-C, Departamento de Ciências Fisiológicas-CCB, Universidade Estadual de Londrina.

*Autor para correspondência. E-mail: damzaia@uel.br 


\section{Introdução}

A química prebiótica estuda as reações e os processos que podem ter desempenhado um papel importante para origem da vida em nosso planeta. Portanto é importante conhecer quais foram as principais fontes de biomoléculas (aminoácidos, lipídios, açúcares, bases nitrogenadas, etc) assim como os biopolímeros (peptídeos, proteínas, DNA, RNA, etc) e as estruturas coacervadas (estruturas parecidas com células) que poderiam ter sido sintetizados na Terra prebiótica (ZAIA, 2003; ZAIA; ZAIA, 2008; ZAIA; ZAIA; DE SANTANA, 2008).

Tiocianato e amônio foram sintetizados em condições utilizadas em experimentos de química prebiótica ou foram encontrados em ambientes que podem ter existido na Terra prebiótica. Tiocianato foi determinado em ambientes hidrotermais e amônio pode ser sintetizado a partir da redução do nitrito pelo Fe (II) (DOWLER; INGMANSON, 1979; SUMMERS, 1999). Tiocianato de amônio foi também sintetizado em condições que reproduzem aquelas da Terra primitiva (RAULIN; TOUPANCE, 1977). Portanto tiocianato e amônio foram substâncias que poderiam ter sido facilmente encontradas na Terra primitiva.

A reação entre o amônio e o tiocianato foi estudada por diversos autores, sendo que algumas das condições utilizadas podem ter existido na Terra prebiótica (STEINMAN; SMITH; SILVER, 1968; ZAIA et al., 2004). As seguintes substâncias foram sintetizadas utilizando o tiocianato e o amônio: enxofre coloidal (HOLMES, 1926), tiouréia (TALREJA; OZA; RAO, 1967; ZAIA, et al., 2004), tiocianato de guanidina (ZAIA, et al., 2004), metionina (STEINMAN; SMITH; SILVER, 1968) e estruturas coacervadas (SMITH; SILVER; STEINMAN, 1968; SMITH; STEINMAN; GALAND, 1969).

No presente trabalho foi estudada a reação entre tiocianato de amônio e Fe (II) ou Fe (III) em estado sólido. As amostras foram aquecidas a $220{ }^{\circ} \mathrm{C} \mathrm{em}$ diversos tempos (6 horas, 24 horas ou 7 dias). $\mathrm{O}$ aquecimento em estado sólido do tiocianato de amônio mais Fe (II) ou Fe (III) foi reproduzido em laboratório como o calor gerado pelo derrame de lava por vulcões na Terra prebiótica ou o impacto de meteoros ou cometas na superfície da Terra prebiótica.

\section{Procedimento Experimental}

\section{Preparo das amostras}

As amostras foram preparadas utilizando 1,00 $\mathrm{g}$ de tiocianato de amônio com 1,00 g sulfato ferroso $\left(\mathrm{FeSO}_{4}\right)$ ou $1,00 \mathrm{~g}$ cloreto férrico $\left(\mathrm{FeCl}_{3}\right)$. As amostras foram submetidas à um pré aquecimento de $110^{\circ} \mathrm{C}$ por 2 horas, e em seguida a temperatura foi aumentada para $220^{\circ} \mathrm{C}$ e as amostras foram aquecidas por 6 horas, 24 horas e 7 dias.

\section{Preparo dos complexos}

Foram preparados os seguintes complexos: sulfato ferroso/tiocianato de amônio ou tiouréia ou tiocianato de guanidina e cloreto férrico/ tiocianato de amônio ou tiouréia ou tiocianato de guanidina. Para o preparo dos complexos foram dissolvidos 0,10 mol de cada substância (tiocianato de amônio, tiouréia, tiocianato de guanidina, sulfato ferroso, cloreto férrico) em $50 \mathrm{~mL}$ de água destilada. Posteriormente $10 \mathrm{~mL}$ de cada sal de ferro foram misturados com $10 \mathrm{~mL}$ de tiocianato de amônio ou tiouréia ou tiocianato de guanidina. As amostras foram mantidas em repouso por 20 minutos e foram posteriormente congeladas e liofilizadas para posterior obtenção dos espectros infravermelhos.

\section{Espectroscopia no infravermelho}

Os espectros foram obtidos a partir de pastilhas de $\mathrm{KBr}$ utilizando o equipamento FT-IR 8300 da Shimadzu, foi utilizado a resolução espectral $4 \mathrm{~cm}-1$ e cada espectro foi obtido após 98 scans. Por volta de $10 \mathrm{mg}$ de amostra mais $200 \mathrm{mg}$ de $\mathrm{KBr}$ foram 
pesados e transferidos para um almofariz de ágata e homogeneizados. As pastilhas foram preparadas e cada espectro foi obtido de 400 a $4000 \mathrm{~cm}^{-1}$. Os espectros foram analisados utilizando o programa Origin $(5.0,2001)$.

\section{Resultados e discussão}

A figura 1 mostra os espectros do tiocianato de amônio, tiouréia, tiocianato de guanidina e ferricianeto de potássio puros, os espectros dos complexos tiocianato de amônio, tiouréia e tiocianato de guanidinina com $\mathrm{Fe}$ (II) ou Fe (III) e os produtos de reação entre Fe (II) ou Fe (III) e tiocianato de amônio aquecidos por 6 horas, 24 horas e sete dias. A figura $1 \mathrm{~A}$ mostra os espectros dos sólidos puros tiocianato de amônio A-a, tiouréia A-b e tiocianato de guanidina A-c. ). As bandas em 2053, 1657 (fraca) e $1402 \mathrm{~cm}^{-1}$, observadas na Figura 1 A-a do espectro do tiocianato de amônio sólido, são atribuídas ao estiramento assimétrico $(\mathrm{C} \equiv \mathrm{N})$ e estiramento $\mathrm{C}=\mathrm{N}$ do grupo $\mathrm{SCN}$ e deformação $\mathrm{NH}_{4}^{+}$, respectivamente (COLTHUP; DALY; WIBERLEY, 1964). As bandas em 1620, 1589 e 1469/1414 $\mathrm{cm}^{-1}$ observadas na Figura 1 A-b do espectro da tiouréia sólido, podem ser atribuídas a deformação simétrica de $\mathrm{NH}_{2}$, deformação assimétrica de $\mathrm{NH}_{2}$ e estiramento $\mathrm{CN}$, respectivamente (BENCIVENNI; NUNZIANTE; PIERETTI, 1998). Observamos também uma banda em $730 \mathrm{~cm}^{-1}$ que pode ser atribuída ao estiramento $\mathrm{C}=\mathrm{S}$ (BENCIVENNI; NUNZIANTE; PIERETTI, 1998) (espectro não mostrado). No caso do tiocianato de guanidina (Figura 1-A-c) a banda em $1656 \mathrm{~cm}^{-1}$ pode ser atribuída ao estiramento $\mathrm{C}=\mathrm{N}($ DROZD, 2008) e as bandas em 2087/2072 $\mathrm{cm}^{-1}$ podem ser atribuídas ao estiramento $\mathrm{C} \equiv \mathrm{N}$ (COLTHUP; DALY; WIBERLEY, 1964).

A figura 1-B mostra os espectros dos complexos de Fe (II) com tiocianato de amônio, tiouréia e tiocianato de guanidina. $\mathrm{O}$ espectro do complexo $\mathrm{Fe}$ (II) com tiocianato de amônio (Figura 1-B-a) mostra uma nova banda em $1423 \mathrm{~cm}^{-1}$ sendo que para as outras bandas não observamos deslocamentos quando comparamos com o espectro do tiocianato de amônio puro (Figura 1-A-a). Esta nova banda pode ser uma indicação da reação entre amônio e Fe (II), visto que em $1400 \mathrm{~cm}^{-1}$ tem-se a deformação do $\mathrm{NH}_{4}^{+}$. Para o complexo entre o Fe (II) e a tiouréia observamos as bandas em 1625, 1473 e $1402 \mathrm{~cm}^{-1}$. Comparando o espectro do complexo Fe (II)/tiouréia com o espectro da tiouréia sólida observamos algumas mudanças sendo estas: desaparecimento da banda em $1589 \mathrm{~cm}^{-1}$ devido ao estiramento assimétrico do $\mathrm{NH}_{2}$ da tiouréia sólida e deslocamentos das bandas devido à deformação simétrica do $\mathrm{NH}_{2}$ e ao estiramento $\mathrm{CN}$. A banda em $730 \mathrm{~cm}^{-1}$ devido ao estiramento $\mathrm{C}=\mathrm{S}$ não foi deslocada, mas observamos uma nova banda em $710 \mathrm{~cm}^{-1}$. Portanto podemos dizer que a interação entre o $\mathrm{Fe}$ (II) e a tiouréia ocorre através dos grupos $\mathrm{NH}_{2}$ da tiouréia. No caso do complexo Fe (II)/ tiocianato de guanidina observamos as bandas em 2115, 2062 (ombro) e $1660 \mathrm{~cm}^{-1}$. A banda em 2062 $\mathrm{cm}^{-1}$ é atribuída ao estiramento $\mathrm{C} \equiv \mathrm{N}$ e a banda em 1660 é atribuída ao estiramento $\mathrm{C}=\mathrm{N}$. A nova banda em $2115 \mathrm{~cm}^{-1}$ também é observada no composto ferricianeto (Figura 1-D-d). Isto pode ser uma indicação da ocorrência de uma reação de oxidação do Fe (II) para Fe (III) pelo tiocianato de guanidina com a formação de um complexo parecido com ferricianeto.

A figura 1-C mostra os espectros dos produtos de reação entre o $\mathrm{Fe}$ (II) e o tiocianato de amônio na proporção de $1,00 \mathrm{~g}: 1,00 \mathrm{~g}$, aquecidos a $220{ }^{\circ} \mathrm{C}$ em diferentes tempos (6,0 h, 24 h, 7 dias). As amostras de tiocianato de amônio/Fe (II) aquecidas por 7 dias, $24 \mathrm{~h}$ e $6 \mathrm{~h}$ a $220^{\circ} \mathrm{C}$ mostraram as seguintes bandas: 2114, 2072, 1658, 1431 e $1401 \mathrm{~cm}^{-1} ; 2114,2072$, 1660, 1641 e $1427 \mathrm{~cm}^{-1}$ e 2114, 2072, 1733 (ombro), 1663 e $1432 \mathrm{~cm}^{-1}$, respectivamente. Portanto podemos inferir que o tempo de aquecimento não tem uma grande influência na reação entre Fe (II) e o tiocianato de amônio. As bandas nas regiões de 2072 e $1658-1663 \mathrm{~cm}^{-1}$ e $2114 \mathrm{~cm}^{-1}$ que aparecem nas amostras aquecidas são características da 


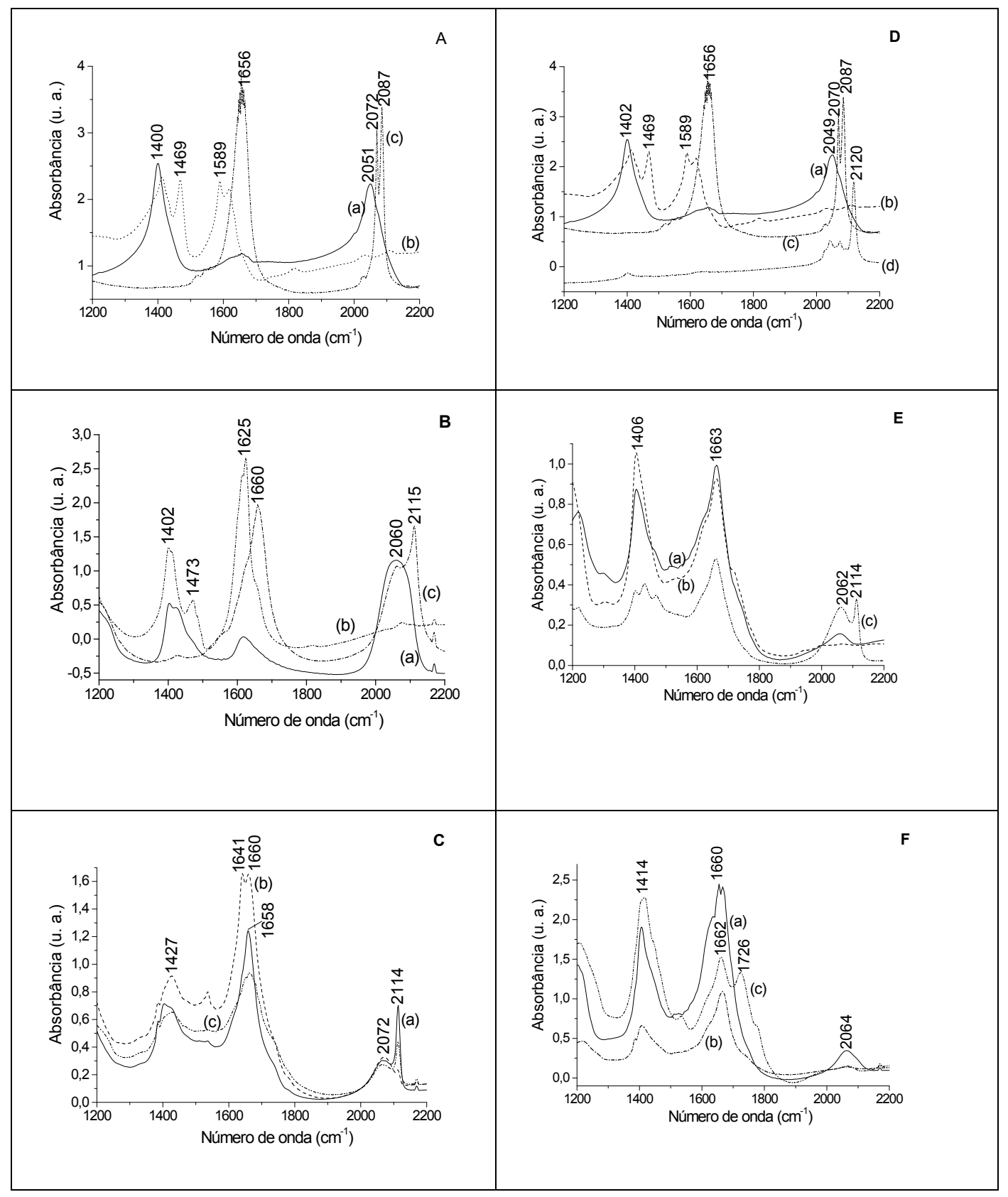

Figura 1. Espectros FT - IR: (A) tiocianato de amônio (a), tiouréia (b) e guanidinina (c), (B) complexos tiocianato de amônio com Fe (II) (a), tiouréia com Fe (II) (b) e guanidinina com Fe (II) (c), (C) reação entre $\mathrm{Fe}(\mathrm{II})$ e tiocianato de amônio aquecido por 7 dias na proporção de 1,000 $\mathrm{g}: 1,000 \mathrm{~g}$ (a), reação entre $\mathrm{Fe}(\mathrm{II})$ e tiocianato de amônio aquecido por 24 horas na proporção de 1,000 g:1,000 g (b); reação entre Fe(II) e tiocianato de amônio aquecido por 6 horas na proporção de 1,000 g:1,000 g (c), (D) tiocianato de amônio (a), tiouréia (b), guanidinina (c) e ferricianeto de potássio (d), (E) complexos tiocianato de amônio com Fe (III) (a), tiouréia com Fe (III) (b) e guanidinina com Fe (III) (c) e (F) reação entre Fe(III) e tiocianato de amônio aquecido por 7 dias na proporção de 1,000g:1,000g (a), reação entre Fe(III) e tiocianato de amônio aquecido por 24 horas na proporção de $1,000 \mathrm{~g}: 1,000 \mathrm{~g}$ (b); reação entre $\mathrm{Fe}(\mathrm{III})$ e tiocianato de amônio aquecido por 6 horas na proporção de 1,000 g:1,000 g (c). Para o preparo dos complexos foi dissolvido 0,10 mol de cada substância em $50 \mathrm{~mL}$. A temperatura utilizada para o aquecimento das amostras foi $220^{\circ} \mathrm{C}$. 
guanidina (Figura 1-A-c) e do complexo Fe (II)/ guanidina (Figura 1-B-c) respectivamente. Portanto o aquecimento pode estar levando a formação guanidina que pode estar sendo complexado com Fe (II). O espectro do ferricianeto mostra uma banda em $2120 \mathrm{~cm}^{-1}$ (Figura 1-D-d). Portanto, a banda observada em $2114 \mathrm{~cm}^{-1}$ poderia também ser característica do estiramento $\mathrm{C} \equiv \mathrm{N}$ quando o cianeto está ligado a Fe (III), sendo está banda é utilizada para medir o estado de oxidação do $\mathrm{Fe}$, visto que quando cianeto reage com Fe (II) está mesma banda é observada numa região de $80 \mathrm{~cm}^{-1}$ abaixo do ponto onde cianeto reage com Fe (III) (LE CAËR et al., 2006). Portanto o aquecimento do tiocianato de amônio com Fe (II) pode estar formando tiocianato de guanidina e ao mesmo tempo o Fe (II) esteja sendo oxidado para Fe (III).

A figura 1-E mostra os espectros dos complexos de Fe (III) com tiocianato de amônio, tiouréia e tiocianato de guanidina. A figura 1-E-a mostra o espectro do complexo formado entre o $\mathrm{Fe}$ (III) e o tiocianato de amônio. Neste espectro observamos bandas nas regiões 2062, 1663 e $1406 \mathrm{~cm}^{-1}$. A banda em $1406 \mathrm{~cm}^{-1}$ foi observada no espectro do tiocianato de amônio (Figura 1-A-a) e é atribuída à deformação $\mathrm{NH}_{4}^{+}$. A banda em $2062 \mathrm{~cm}^{-1}$ mostra um deslocamento da observada no espectro do tiocianato de amônio (2053 $\left.\mathrm{cm}^{-1}\right)$, esta banda foi atribuída ao estiramento assimétrico $(\mathrm{C} \equiv \mathrm{N})$ do grupo $\mathrm{SCN}$ (COLTHUP; DALY; WIBERLEY, 1964). A banda em $748 \mathrm{~cm}^{-1}$ atribuída ao estiramento simétrico do íon tiocianato foi deslocada para $773 \mathrm{~cm}^{-1}$ sendo assim o metal está coordenando com o nitrogênio do íon tiocianato (MNCS) (COLTHUP; DALY; WIBERLEY, 1964). Portanto estes deslocamentos indicam que o tiocianato está interagindo com o Fe (III). O aumento da intensidade da banda em 1663 $\mathrm{cm}^{-1}$ é uma indicação que quando o complexo de ferro (III) tiocianato é formado os elétrons da tripla ligação $\mathrm{C} \equiv \mathrm{N}$ são deslocados favorecendo a formação de $\mathrm{C}=\mathrm{N}$. Este efeito não foi observado quando foi preparado o complexo Fe (II) tiocianato de amônio (Figura 1-B-a). O espectro do complexo Fe (III) com tiouréia tem as seguintes bandas 1718 (ombro), 1663 e $1406 \mathrm{~cm}^{-1}$ (Figura 1-E-b). Comparando o espectro do complexo Fe (III)/tiouréia (Figura 1-E-b) com o espectro da tiouréia sólida (Figura 1-D-b), verificamos que as bandas em 1620, 1589, 1469 e $730 \mathrm{~cm}^{-1}$ devido a deformação simétrica e assimétrica do $\mathrm{NH}_{2}$ e aos estiramentos $\mathrm{CN}$ e $\mathrm{C}=\mathrm{S}$ da tiouréia sólida (BENCIVENNI, NUNZIANTE, PIERETTI, 1998) desapareceram no complexo Fe (III)/tiouréia. No complexo Fe (III)/tiouréia duas novas bandas foram observadas sendo estas em 1718 (ombro) e $1663 \mathrm{~cm}^{-1}$. Estas mudanças no espectro do complexo $\mathrm{Fe}$ (III)/tiouréia estão indicando que o Fe (III) esta interagindo com tiouréia através do grupo $\mathrm{C}=\mathrm{S}$, isto pode ser comprovado visto que ocorreu o desaparecimento da banda em $730 \mathrm{~cm}^{-1}$ devido ao estiramento $\mathrm{C}=\mathrm{S}$ e uma nova banda em $770 \mathrm{~cm}^{-1}$ devido ao estiramento C-S foi observada. De Santana et al. (2009) estudaram a reação entre tiouréia e argilas as quais contém Fe (III) e observaram a formação do íon ditiodiformamida $\{[\mathrm{NH} 2(\mathrm{NH}) \mathrm{CSSC}(\mathrm{NH}) \mathrm{NH} 2] 2+\}$. A figura 1-E-c mostra o espectro do complexo Fe (III)/tiocianato de guanidina sendo que as seguintes bandas foram observadas: 2114, 2062, 1660, 1470, 1432 e 1401 $\mathrm{cm}^{-1}$. As bandas em 2114, 2062 e $1660 \mathrm{~cm}-1$ foram também observadas no complexo Fe (II)/tiocianato de guanidina (Figura 1-B-c). No entanto não foi possível relacionar as outras bandas $(1470,1432 \mathrm{e}$ $\left.1401 \mathrm{~cm}^{-1}\right)$ com as bandas das outras moléculas aqui estudadas.

A figura 1-F mostra os espectros dos produtos de reação entre o $\mathrm{Fe}$ (III) e o tiocianato de amônio na proporção de $1,00 \mathrm{~g}: 1,00 \mathrm{~g}$, aquecidos a $220^{\circ} \mathrm{C}$ em diferentes tempos (6,0 h, 24 h, 7 dias). As amostras de tiocianato de amônio/Fe (III) aquecidas por 7 dias, $24 \mathrm{~h}$ e $6 \mathrm{~h}$ a $220{ }^{\circ} \mathrm{C}$ mostraram as seguintes bandas: 2066, 1669, 1654, 1438 (ombro), 1405 e 1386 (ombro) cm $\mathrm{cm}^{-1} ; 2066$ (fraca), 1666, 1407 e 1388 (ombro) $\mathrm{cm}^{-1}$ e 2066 (fraca), 1776 (ombro), 1660, 1628 (ombro), 1448 (ombro), 1414 e 1386 (ombro) $\mathrm{cm}^{-1}$, respectivamente. Novamente observamos que o tempo de aquecimento não influencia na reação 
entre tiocianato de amônio e o Fe (III) em estado sólido. Os espectros dos produtos de reação do $\mathrm{Fe}$ (III) com tiocianato de amônio (Figuras 1-F-a,b,c) são em geral parecidos com o espectro do complexo Fe (III) tiocianato de guanidina. No entanto neste caso não observamos a banda em $2114 \mathrm{~cm}^{-1}$ como foi observado para o caso da reação $\mathrm{Fe}$ (II) tiocianato de amônio em estado sólido (Figura 1-C-a,b,c) ou complexo $\mathrm{Fe}$ (III)/tiocianato de guanidina (Figura 1-E-c) ou mesmo o complexo Fe (II)/tiocianato de guanidina (Figura 1-B-c). Esta reação está formando o tiocianato de guanidina, no entanto o produto final não é o mesmo.

\section{Conclusões}

Uma reação de oxidação-redução pode estar ocorrendo entre o Fe (II) e o tiocianato de guanidina, visto que este complexo apresenta uma banda no infravermelho que é característica do ferricianeto.

$\mathrm{O}$ aquecimento do tiocianato de amônio com $\mathrm{Fe}$ (II) pode estar formando tiocianato de guanidina e ao mesmo tempo oxidando o Fe (II) para Fe (III).

$\mathrm{A}$ interação entre $\mathrm{Fe}$ (III) e tiocianato de amônio e tiouréia é através dos grupos $\mathrm{C}=\mathrm{N}$ e $\mathrm{C}=\mathrm{S}$, respectivamente.

O produto da reação entre $\mathrm{Fe}$ (III) e tiocianato de amônio é o tiocianato de guanidina.

$\mathrm{O} F$ (II) e o Fe (III) reagem de forma diferente com o tiocianato de amônio.

A reação entre o $\mathrm{Fe}$ (II) e o tiocianato de amônio pode ter desempenhado um papel importante na química prebiótica visto que ocorreu a formação de tiocianato de guanidina e ao mesmo tempo gerou $\mathrm{Fe}$ (III). O Fe (III) e o tiocianato de guanidina desempenham importantes papeis nos seres vivos atuais.

\section{Referências}

BENCIVENNI, L.; NUNZIANTE, C.; PIERETTI, A. Matrix and ab initio infrared spectra of thiourea and thiourea-d4. Vibrational Spectroscopy, Amsterdam, v. 18, n. 2, p. 91-102, 1998.

COLTHUP, N. B.; DALY, L. H.; WIBERLEY, S. E. Introduction to infrared and raman spectroscopy. New York: Academic Press, 1964.

DE SANTANA, H.; PAESANO JUNIOR, A.; DA COSTA, A. C. S.; DI MAURO, E.; DE SOUZA, I. G.; IVASHITA, F. F.; DE SOUZA, C. M. D.; ZAIA, C. T. B. V.; ZAIA, D. A. M. Cysteine, thiourea and thiocyanate interactions with clays: FT-IR, Mössbauer and EPR spectroscopy and X-ray diffractometry Studies. 2009. Disponivel em: <http://www.springerlink.com/content/ k872u10753866144/fulltext.pdf $>$. Acesso em: 11 mar. 2010.

DOWLER, M. J.; INGMANSON, D. E. Thiocyanate in Red Sea brine and its implications. Nature, London v. 279, p. 51-52, 1979.

DROZD, M. New complexes of guanidine with acetic, trichloroacetic and trifluoroacetic acids the DFT structural and vibrational investigations. Spectrochimica Acta Part A, Oxford, v. 69, n. 4, p. 1223-1234, 2008.

HOLMES, M. The action of light on concentrated aqueous solutions of ammonium thiocyanate. Journal of the Chemical Society, London, p. 1690-1693, 1926.

LE CAËR, S.; VIGNERON, G.; RENAULT, J. P.; POMMERET, S. First coupling between a LINAC and FT-IR spectroscopy: the aqueous ferrocyanide system. Chemical Physics Letters, Amsterdam, v. 426, n. 1/3, p. 71-76, 2006

RAULIN, F.; TOUPANCE, G. The role of sulphur in chemical evolution. Journal of Molecular Evolution, New York, v. 9, n. 4, p. 329-338, 1977.

SMITH, A. E.; SILVER, J. J.; STEINMAN, G. Celllike structures from simple molecules under simulated primitive earth conditions. Cellular and Molecular Life Sciences, Basel, v. 24, n. 1, p. 36-38, 1968.

SMITH, A. E.; STEINMAN, G.; GALAND, C. Possible abiotic origin of precambrian microfossils. Cellular and Molecular Life Sciences, Basel, v. 25, n. 3, p. 255-258. 1969.

STEINMAN, G.; SMITH, A. E.; SILVER, J. J. Synthesis of a sulfur-containing amino acid under simulated prebiotic conditions. Science, Washington, v. 159, n. 819, p. 1108-1109, 1968. 
SUMMERS, D. P. Sources and sinks for ammonia and nitrite on the early earth and the reaction of nitrite with ammonia. Origins of Life and Evolution of the Biosphere, Dordrecht, v. 29, n. 1, p. 33-46, 1999.

TALREJA, S. T.; OZA, P. M.; RAO, P. S. Radiation induced conversion of ammonium thiocyanate to thiourea. Bulletin of the Chemical Society of Japan, Tókio, v. 40, n. 10, p. 2427-2428, 1967.

ZAIA, D. A. M. Da geração espontânea à química prebiótica. Química Nova, São Paulo, v. 26, n. 2, p. 260 264, 2003.

ZAIA, D. A. M.; DE SANTANA, H.; TOPPAN, R.; ZAIA, C. T. B. V. Synthesis of guanidine from ammonium thiocyanate in solid state. Journal of the
Brazilian Chemical Society, São Paulo, v. 15, n. 2, p. 190-198, 2004.

ZAIA, D. A. M.; ZAIA, C. T. B. V. Algumas controvérsias sobre a química prebiótica. Química Nova, São Paulo, v. 31, n. 1, p. 1599-1602, 2008.

ZAIA, D. A. M.; ZAIA, C. T. B. V.; DE SANTANA, H. Which amino acids should be used in prebiótica chemistry studies? Origins of Life and Evolution of the Biosphere, Dordrecht, v. 38, n. 1, p. 469-488, 2008.

Recebido em 17 Dezembro, 2009 - Received on December 17, 2009. Aceito em 9 Março, 2010 - Accepted on March 9, 2010. 